Article

\title{
Ultra-Low Reflectivity Anti-Reflection Coating on a Plastic Cover Slip in Liquid for He-Ne Laser Light
}

\author{
Jin-Cherng Hsu ${ }^{1,2, *}$, Heng-Ying Cho ${ }^{1}$, Tsang-Yen Hsieh ${ }^{3}$ and Jyh-Liang Wang ${ }^{3}$ \\ 1 Department of Physics, Fu Jen Catholic University, New Taipei City 24205, Taiwan; k00165105@hotmail.com \\ 2 Graduate Institute of Applied Science and Engineering, Fu Jen Catholic University, \\ New Taipei City 24205, Taiwan \\ 3 Department of Materials Engineering, Ming Chi University of Technology, New Taipei City 24301, Taiwan; \\ tyhsieh@mail.mcut.edu.tw (T.-Y.H.); joewang@mail.mcut.edu.tw (J.-L.W.) \\ * Correspondence: 054326@mail.fju.edu.tw; Tel.: +886-2-2905-3765; Fax: +886-2-2902-1038
}

Received: 31 May 2017; Accepted: 26 June 2017; Published: 29 June 2017

\begin{abstract}
An ultra-low anti-reflection optical coating on both surfaces of a plastic cover slip was studied for use in confocal image measurements. The optical reflectance at a wavelength of $632.8 \mathrm{~nm}$ was less than $0.1 \%$ when the coated sample was placed in a liquid having a refractive index of 1.34 , close to the aqueous solution of the biomaterial. The high- and low-index coating films, Substance-2 $\left(\mathrm{PrTiO}_{3}\right)$ and silicon dioxide $\left(\mathrm{SiO}_{2}\right)$, were measured by an ellipsometer to determine their optical refraction indices and extinction coefficients. Theoretically, when the two layer thicknesses are designed using the optical admittance diagram of the cover slip to approach the equivalent index of 1.34 , a reflectance of $1.6 \times 10^{-5} \%$ in the liquid could be obtained. Experimentally, the reflectance of the sample deposited on the two faces of the cover slip was $4.223 \pm 0.145 \%$ as measured in the air; and $0.050 \pm 0.002 \%$ as measured by a He-Ne laser in the liquid.
\end{abstract}

Keywords: ultra-low anti-reflection coating; ellipsometer; optical admittance method

\section{Introduction}

An anti-reflection coating process is usually a significant treatment for the surface of an optical element. It can increase the optical transmission and decrease optical disturbances from the optical reflection of the element's surface. Optical disturbances are largely enhanced when optical elements are illuminated by a laser beam, since laser beam has a small dispersion angle, a high power density, excellent coherence properties, etc. A confocal microscopy system is usually equipped with the laser beam due to the high power density because the system contains two pinholes to mainly limit the illumination light. However, when the laser irradiates a biological sample, the good coherence of the beam tends to cause interference between the two surfaces of the cover sheet, which may reduce the resolution of the biological image [1]. Moreover, the reflected light from the liquid and the cell interface of the sample may interfere with other light generated at the interface of the coverslip liquid. Thus, the image intensity is affected by constructive or destructive interference [2]. In order to avoid the influence of the optical interference of the laser beam during confocal measurements, the treatment of both surfaces of a plastic cover slip with an ultra-low anti-reflection coating at a wavelength of $632.8 \mathrm{~nm}$ is necessary when the confocal microscopy system uses a He-Na laser as the illumination light source. The coating materials, the number of layers, and film thicknesses must be designed for use in the optical coating due to a large amount of heat generated during the optical coating process in the deposition vacuum chamber and the poor heat resistance of the plastic substrate. 


\section{Materials and Methods}

\subsection{Optical Analysis of the Material}

For the ultra-low anti-reflection coating, the optical constants of the deposited substrate and optical films were precisely measured as follows. The substrate (Thermanox Coverslip-174977, Thermo Fisher Scientific Inc., Waltham, MA, USA), which was made of polystyrene and had a diameter of $22 \mathrm{~mm}$ and a thickness of $0.2 \mathrm{~mm}$, was ultrasonically cleaned for $15 \mathrm{~min}$ and then dried with an air spray gun. The transmittance was measured using a Varian Cary 5E spectrophotometer (Varian, Palo Alto, CA, USA) at wavelengths of $400 \mathrm{~nm}$ to $700 \mathrm{~nm}$ in the visible spectrum. To measure the precise optical constants of the plastic substrate, the back surface of the plastic substrate was carefully ground and blackened to eliminate its reflection. The polarized light reflected by the front surface in the visible area at three incident angles of $55^{\circ}, 60^{\circ}$, and $65^{\circ}$ was evaluated using a VASE ellipsometer made by J. A. Woollam Co., Inc. (Lincoln, NE, USA). The two coating materials, silicon dioxide and Substance-2 made by Merck, were respectively deposited on two silicon wafer substrates using a vacuum deposition method and their optical constants were also evaluated using the VASE ellipsometer.

\subsection{Thin Film Design}

In this study, we used the optical admittance method to design the double-layer anti-reflection coating on the plastic substrate. The coating materials for the double layer were silicon dioxide and Substance-2, with a low and a high optical index, denoted by $n_{\mathrm{L}}$ and $n_{\mathrm{H}}$, respectively. The $\mathrm{d} H$ and $\mathrm{d} L$ are the thicknesses of the $n_{\mathrm{H}}$ layer and $n_{\mathrm{L}}$ layer, respectively. The admittance of the substrate is expressed as $Y_{\mathrm{s}}$, where $Y_{\mathrm{s}}=\left(n_{\mathrm{s}}-i k_{\mathrm{s}}\right) Y_{0}$, and $Y_{0}$ is the admittance of free space. When the reference plane moves from the substrate surface to the input surface of the double layer, the input admittance $Y=C / B$ can be calculated at the new reference plane by the characteristic matrix expressed as:

$$
\begin{aligned}
& {\left[\begin{array}{l}
B \prime \\
C \prime
\end{array}\right]=\left[\begin{array}{cc}
\cos \delta_{L} & i \frac{\sin \delta_{L}}{n_{L}} \\
i n_{L} \sin \delta_{L} & \cos \delta_{L}
\end{array}\right]\left[\begin{array}{c}
1 \\
Y_{S}
\end{array}\right]} \\
& {\left[\begin{array}{c}
B \\
C
\end{array}\right]=\left[\begin{array}{cc}
\cos \delta_{H} & i \frac{\sin \delta_{H}}{n_{H}} \\
i n_{H} \sin \delta_{H} & \cos \delta_{H}
\end{array}\right]\left[\begin{array}{c}
1 \\
Y_{\prime}
\end{array}\right]}
\end{aligned}
$$

where,

$$
Y \prime=C \prime / B \prime, \text { and } \delta_{L}=\frac{2 \pi}{\lambda} n_{L} d_{L}
$$

$\delta_{H}$ and $\delta_{L}$ are the phase thicknesses of the silicon dioxide and Substance- 2 films, respectively. Then, the reflectance $R$ of the double-layer film on the substrate is calculated by [3-5]:

$$
R=\left|\frac{Y_{l}-Y}{Y_{l}+Y}\right|^{2}
$$

where $Y_{1}\left(=n_{1} Y_{0}\right)$ is the admittance of the liquid immersing the plastic substrate in our study. The value of the reflectance approaches zero if the equivalent admittance $Y$ equals that of the liquid $Y_{1}$. In this case, the double-layer film exactly matches the optical indices of the plastic substrate $n_{\mathrm{s}}-i k_{\mathrm{s}}$ and of the liquid $n_{1} 1.34$, which is a compound of ethylene glycol and pure water. The index of the liquid is close to the index of the biological material aqueous solution, allowing for strong optical coupling for biomedical applications [6]. The optical index of the liquid was confirmed by an Abbe refractometer. For the purpose of anti-reflection matching, we then designed a LabVIEW program (version 7.1, National Instruments, TX, USA) to find the correct thickness of the double layer using the graphical technique of the admittance diagram [3]. 


\subsection{Deposition of the Thin Film}

The index of each layer should be precisely determined by the coating process. At first, the substrates were ultrasonically cleaned in pure water for $15 \mathrm{~min}$, dried with an air spray gun, and then put in the substrate holder of an $80 \mathrm{~cm}$ diameter vacuum chamber. The required base pressure for evaporation $\left(6.0 \times 10^{-6}\right.$ torr $)$ was obtained using a diffusion pumping system. A silicon dioxide film was prepared by thermal evaporation of the silicon monoxide granule from a molybdenum evaporation boat and a Substance-2 film was prepared by E-gun evaporation with argon ion-assisted deposition, to evaluate the optical constants of the two films deposited on the silicon wafer substrates, respectively. The ion voltage and ion current used in the home-made end-Hall ion source were $100 \mathrm{~V}$ and $0.5 \mathrm{~A}$, respectively. Moreover, oxygen gas was fed at $70 \mathrm{sccm}$ into the chamber to reach a partial pressure of $2.0 \times 10^{-4}$ torr. The deposition rate and the thicknesses of the two films, which were monitored by a quartz monitor during the deposition process, were about $0.1 \mathrm{~nm} / \mathrm{s}$ and $100 \mathrm{~nm}$, respectively.

Before applying the double layer coating to the plastic substrate, its surface was cleaned using energetic Ar ions produced by the ion source in a vacuum for $10 \mathrm{~min}$. According to the evaluation of the optical constants, the silicon dioxide and Substance- 2 films were, in turn, deposited on each surface of the plastic substrates at $71.71 \mathrm{~nm}$ and $11.15 \mathrm{~nm}$, respectively, with the abovementioned deposition process.

\subsection{Optical Measurements}

Before measuring the deposited sample while it was immersed in the liquid, the He-Ne beam was adjusted to the center of a beaker that had a diameter of $10 \mathrm{~cm}$ and was filled with the liquid. The power of the laser beam was measured by a power meter in a dark laboratory to determine a baseline measurement. The laser beam then illuminated the sample at incident angles of $20^{\circ}$ and $45^{\circ}$. The distance between each power meter and the beaker center was $15.5 \mathrm{~cm}$ as shown in Figure $1 \mathrm{a}, \mathrm{b}$ and Figure 2. We fine-tuned the power meter positions to measure the maximum values of the transmission and reflection of the laser beams at the incident angles.

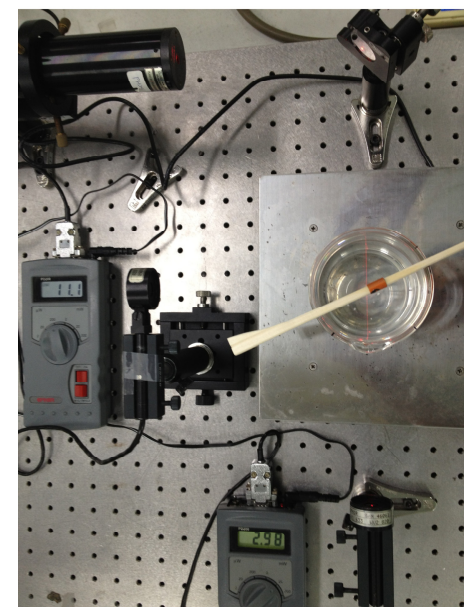

(a)

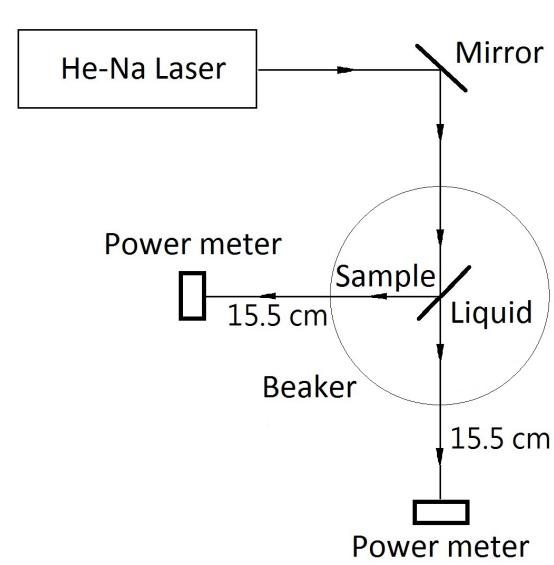

(b)

Figure 1. (a) Picture of the optical system for measuring reflection and transmission; (b) Relative schematic diagram of the optical system. 


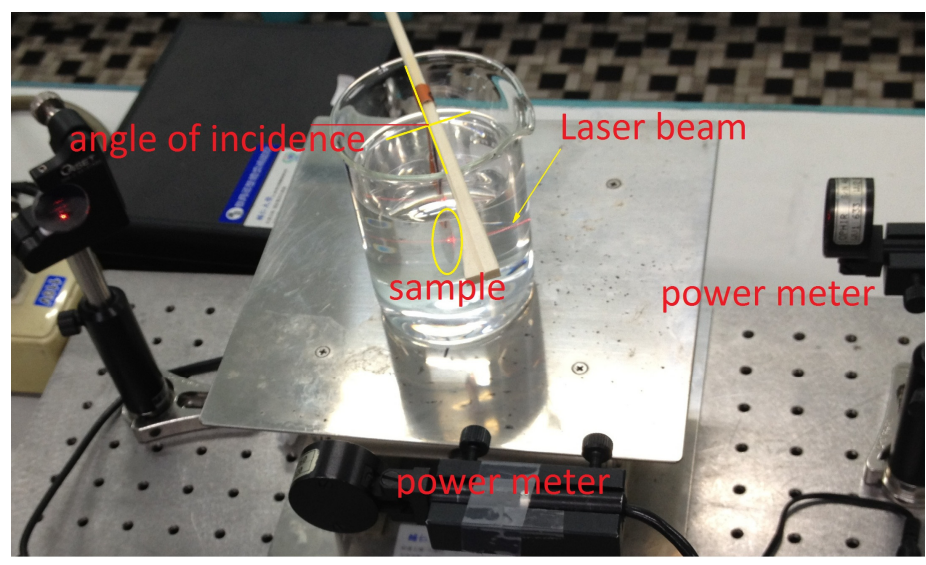

Figure 2. The picture shows the anti-reflection sample measured in the liquid. The blurred red light in the beaker is an optical scattering of the He-Ne laser beam.

\section{Results and Discussion}

The transmittance of the plastic substrate measured by the spectrophotometer was $85.93 \%$ at a wavelength of $632.8 \mathrm{~nm}$, as shown in Figure 3. We found that the substrate had a higher optical refraction index or optical absorption than those of the B270 or PMMA substrates due to the lower transmittance value. Therefore, we carefully measured its optical constants again using the ellipsometer. Figure 4 shows the measured $\psi$ and $\Delta$ data at the three incident angles of $55^{\circ}, 60^{\circ}$, and $65^{\circ}$. The experimental values are unstable due to the weak reflection of the transparent surface of the substrate. However, we constructed a Cauchy model for a single material containing only the optical constants. The surface roughness of the plastic substrate can be neglected because it is too small to trap moisture from the air, and therefore will not have an effect on the ellipsometry measurement [7]. The $\psi$ and $\Delta$ data were simulated by the ellipsometry software WVASE32 provided by J. A. Woollam Co. (Lincoln, NE, USA). The mean squared error (MSE) value of the fitted result is only 1.047 [8], which illustrates that the simulated optical constant of $n_{\mathrm{s}}-i k_{\mathrm{s}}$ is reliable due to the small value. Figure 5 shows the results of the optical index $n_{\mathrm{s}}$ and the extinction coefficient $k_{\mathrm{s}}$, where $n_{\mathrm{S}}$ is 1.73 , and $k_{\mathrm{s}}$ is 0.068 at a wavelength of $632.8 \mathrm{~nm}$.

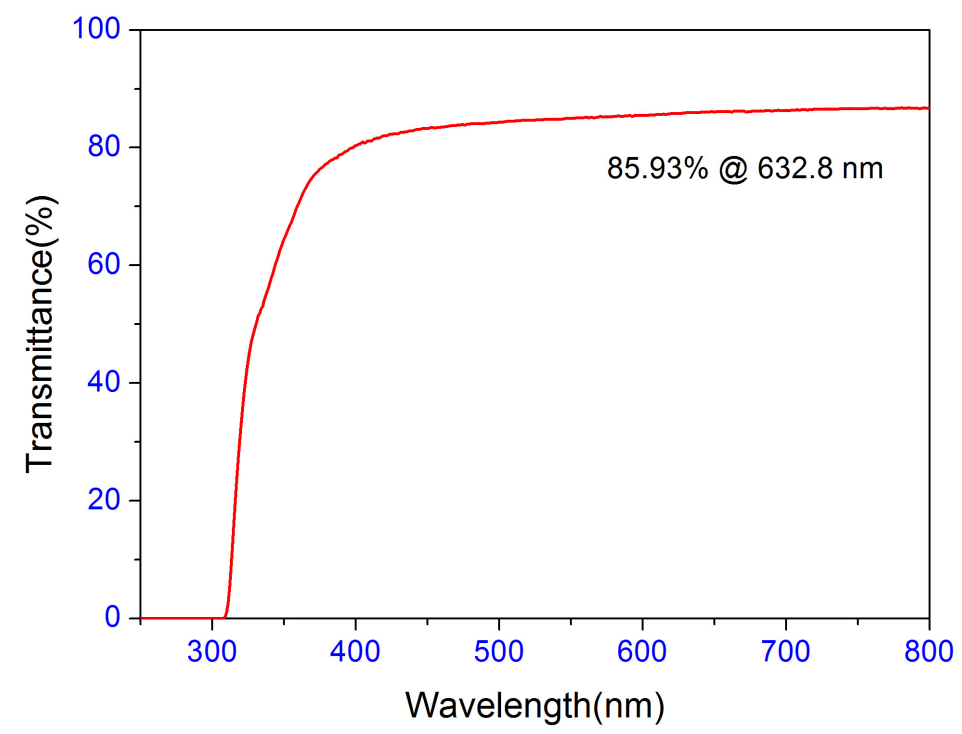

Figure 3. The transmittance of the plastic substrate, where $85.93 \%$ of the value is the transmittance at a wavelength of $632.8 \mathrm{~nm}$. 


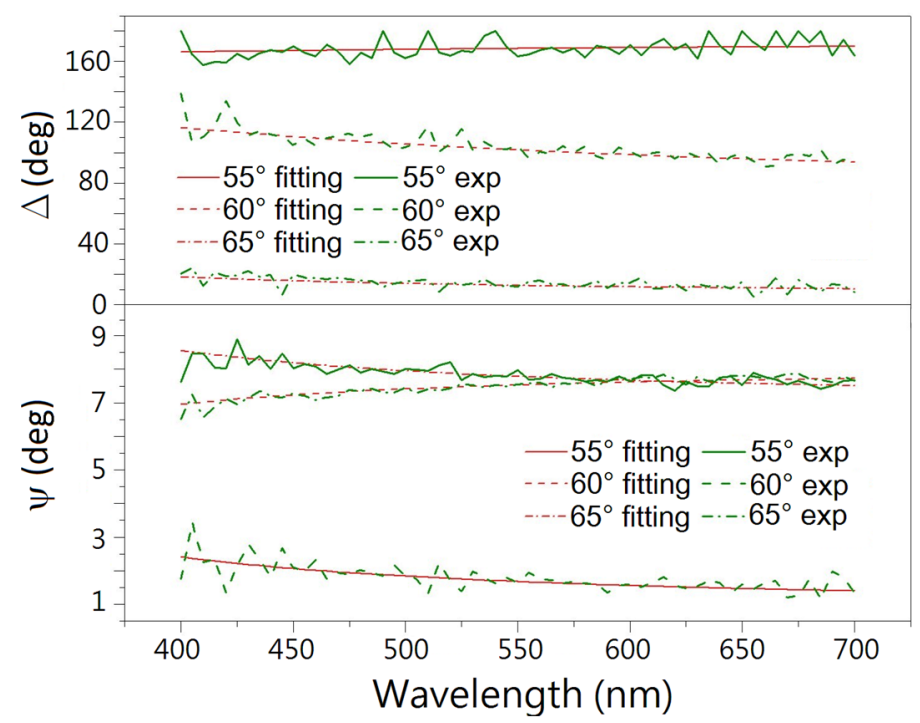

Figure 4. The results of the model fitting: The green line is the experimental data, and the red line is the result of the simulation fitting.

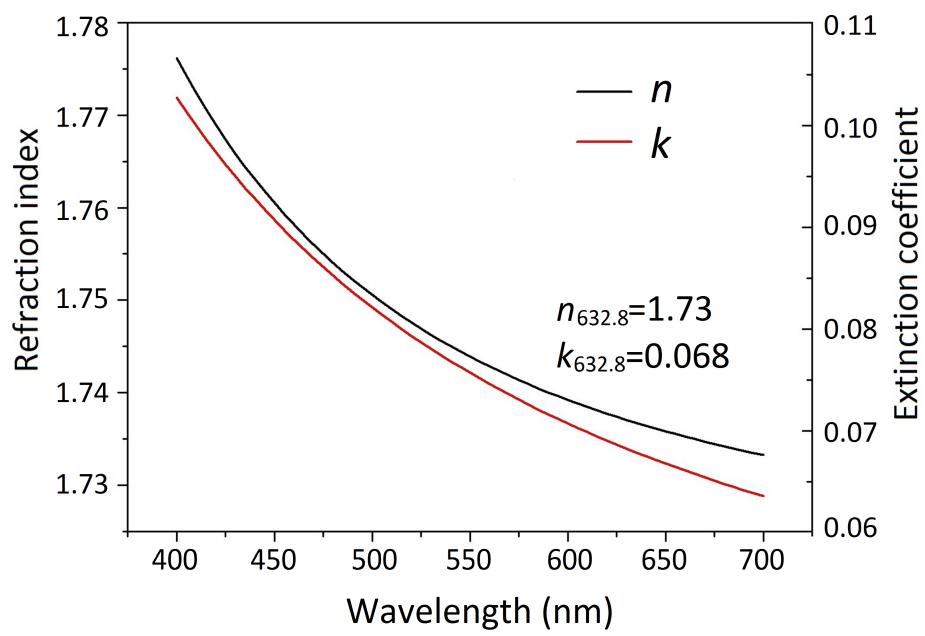

Figure 5. The optical constant of the substrate: The black line is the refractive index $n$, and the red line is the extinction coefficient $k$ in the visible area.

Figure $6 \mathrm{a}, \mathrm{b}$ shows the measured $\psi$ and $\Delta$ data and the simulated results of the silicon dioxide and Substance-2 films on the silicon wafer substrates. We constructed a Cauchy model for a single layer of the film on the substrate, where the surface roughness determined by the substrate was also neglected because the ion assisted deposition during the coating process decreased the boundary scattering and optical absorption, and stabilized the refraction index of the films $[9,10]$. For the measurements of the coating materials, the MSE values of the data fitting results are 2.27 and 2.75, respectively. The two coating materials have a low extinction coefficient $k$ and a suitable refraction index $n$ for the plastic substrate, shown in Figure 7. The silicon dioxide has a low optical index of 1.464 and no optical absorption at a wavelength of $632.8 \mathrm{~nm}$. The other coating material, Substance-2, has a higher optical index of 1.967 and a small extinction coefficient value of $4.3 \times 10^{-4}$, as shown in Figure $7 \mathrm{a}, \mathrm{b}$, respectively. It was found that the Substance- 2 film deposited under non-heated conditions had a slight absorption due to the lower oxidation of the titanium compound [11]. 


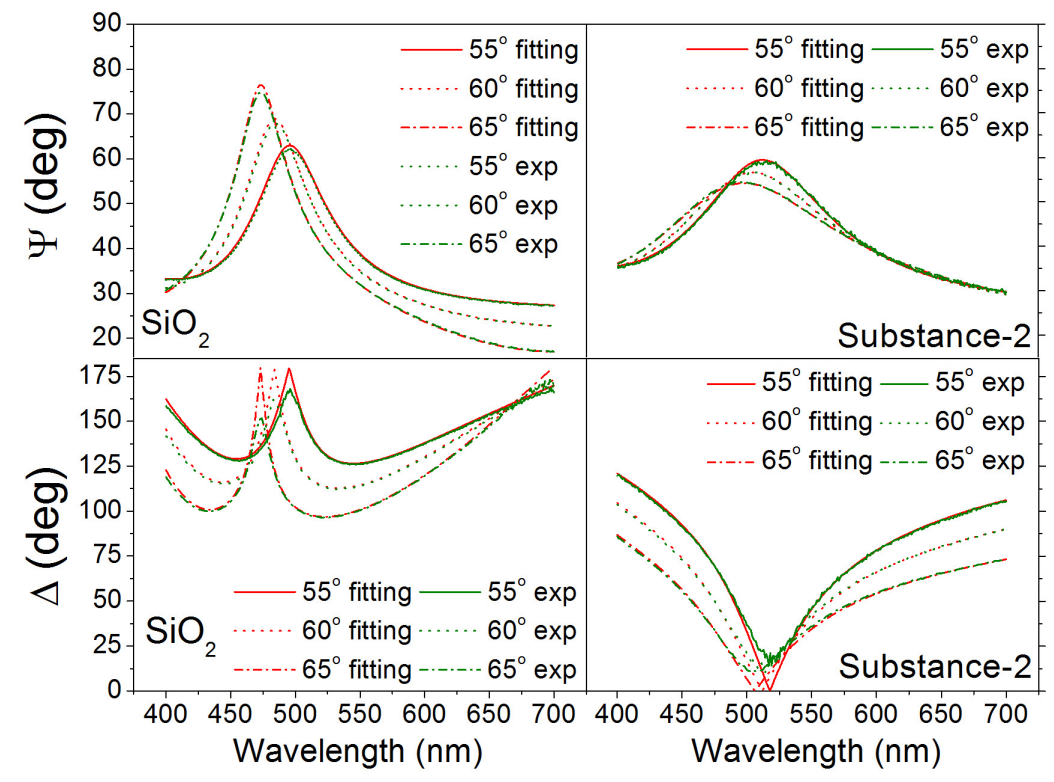

(a)

(b)

Figure 6. The results of the model fitting for (a) silicon dioxide, (b) Substance-2. The green lines are the experimental data, and the red lines are the model fitting results.

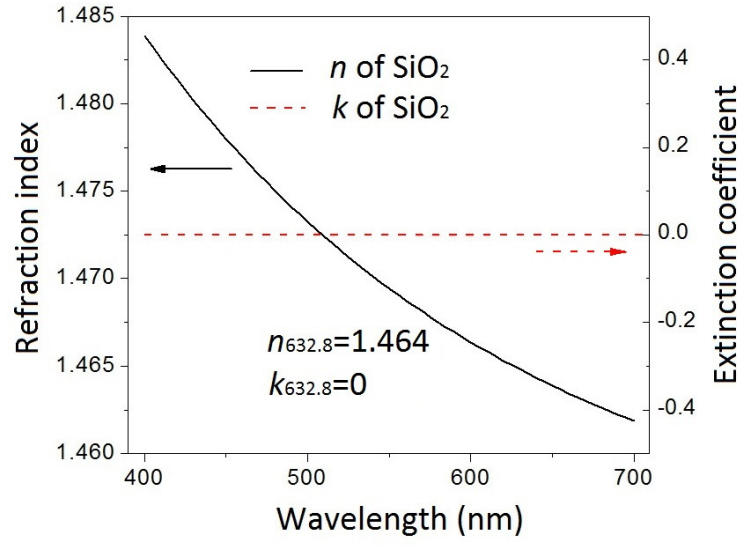

(a)

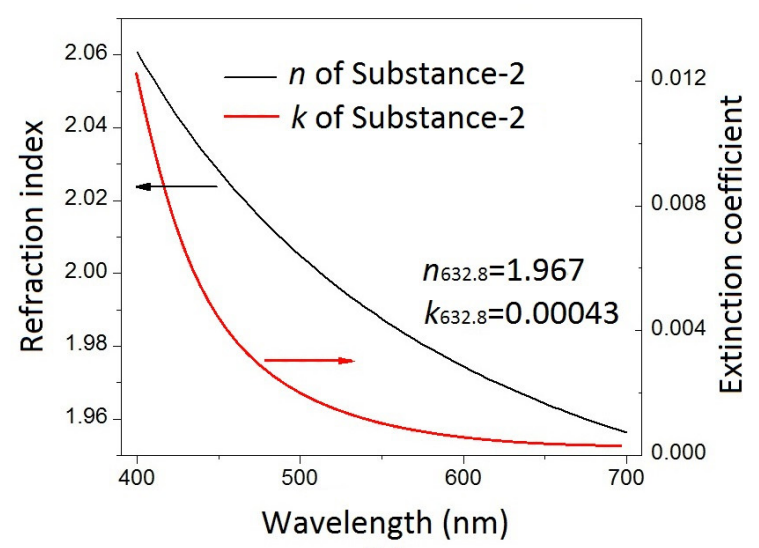

(b)

Figure 7. The optical constants of (a) silicon dioxide (b) Substance-2 in the visible area.

Because the deposited substrate is a plastic material that is easily deformed by the heat during the vacuum deposition, the anti-reflection coating requires a smaller thickness and a smaller amount of deposited film. Catalán has expressed that such an anti-reflection coating can only be designed using a double layer [12]. In this study, we chose silicon dioxide and Substance-2 as the two materials for the double layer. The silicon dioxide was deposited by thermal evaporation with silicon monoxide granules as the starting material [13], because the evaporation temperature of silicon monoxide, $1200-1600{ }^{\circ} \mathrm{C}$, is lower than that of silicon dioxide, $1800-2200{ }^{\circ} \mathrm{C}$.

The thickness of the double-layer was designed using the admittance diagram shown in Figure 8. For the purpose of drawing an admittance diagram, it is convenient to set all optical indices in units of $Y_{0}$. Then, the optical admittance will have the same numerical value as the refractive index. From Equation (2) $Y^{\prime},(x+i y) Y_{0}$, represents the admittance of the double layer at the exit side of the silicon dioxide layer. The equation for the circle of the arc shown in Figure 8 is:

$$
x^{2}+y^{2}-x\left[\left(n_{s}^{2}+K_{s}^{2}+n_{L}^{2}\right) / n_{s}\right] Y_{0}+n_{L}^{2} Y_{0}^{2}=0
$$




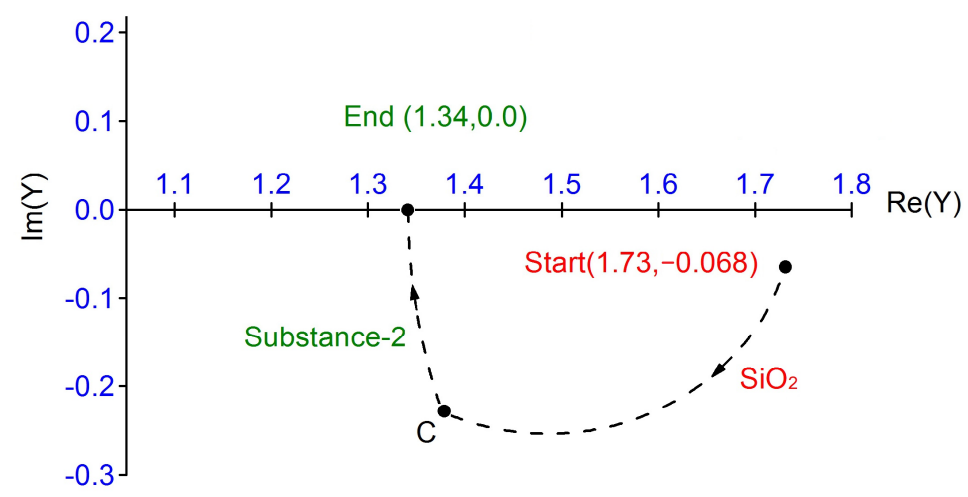

Figure 8. Admittance diagram for the double-layer anti-reflection coating.

Its center is at $\left[\left(n_{\mathrm{s}}^{2}+k_{\mathrm{s}}{ }^{2}+n_{\mathrm{L}}{ }^{2}\right) / 2 n_{\mathrm{s}}, 0\right]$ and it passes through the starting point $\left(n_{\mathrm{s}},-i k_{\mathrm{s}}\right)$ in the complex plane. The circle is followed in the clockwise direction from the starting point. The admittance point $C$ is located when the thickness of the silicon dioxide is increased to the first design thickness of $71.71 \mathrm{~nm}$. This location becomes the starting point of the second circle in the arc, as shown in Figure 8. The second circle of the deposited Substance-2 film is traced clockwise as the layer successively increases from zero thickness to its designed value. As can be seen from the admittance locus, only the locus passes through the end point $(1.34,0)$ to obtain zero reflectance; here the thickness of the second designed film is $11.15 \mathrm{~nm}$. The LabVIEW program can easily try to calculate the thickness of each pair of double-layers, which is based on the exact solution referred to in reference [3]. According to the theoretical calculation above, the equivalent admittance $Y$ was $1.341-i 0.000135$ and the reflectance approaches $1.6 \times 10^{-5} \%$. The presence of the double-layer coating produces about zero reflectance at only one wavelength and a low reflectance limited in a narrow region. However, depositing only double layers benefits the plastic substrate due to the low temperature of its softening point. The first layer is thicker than the second layer, which also reduces the heat generation during the deposition process, because the evaporation temperature of silicon monoxide is much lower than that of Substance-2, at $2000-2200{ }^{\circ} \mathrm{C}$.

In our previous study, it was suggested that the plastic substrate be pretreated with plasma before the deposition and that an oxygen-deficient oxide layer, such as $\mathrm{SiO}_{x}$ (where $x$ is less than and close to 2), be used as a pre-coating layer since the oxygen-deficient oxide tends to chemically bind to plastic substrates [14,15]. Kahler et al. have also observed $\mathrm{SiO}_{x}(1<x<2)$ layers with adjustable oxygen content by thermal evaporation of silicon monoxide in a controlled oxygen atmosphere. The chemical disorder in the matrix for $\mathrm{SiO}_{x}$ is higher than that in a pure silicon dioxide film $[13,16]$. In our study, an amorphous film with a small oxygen deficiency was, therefore, pre-deposited on the plasma-pretreated substrate at a low deposition rate with ion-assisted deposition in a non-heated process. These enhance the adhesion of the boundary between the first deposited film and the plastic substrate. Moreover, the evaporation temperature of the second layer Substance- 2 is much lower than that of the other metal-oxide coating materials that have a high refraction index. The options described above are the best choice for the plastic substrate coatings. Finally, the ultra-low anti-reflection coating was designed and deposited on the sample, as shown in Figure 9, to eliminate the laser-beam reflection from the two surfaces. 


\begin{tabular}{clll} 
Liquid & $n=1.34$ & & \\
\hline Substance-2 & $n=1.967$ & $k=0.00043$ & $d=11.15 \mathrm{~nm}$ \\
\hline $\mathrm{SiO}_{2}$ & $n=1.464$ & $k=0$ & $d=71.71 \mathrm{~nm}$ \\
\hline Plastic Substrate & $n=1.73$ & $k=0.068$ & $d=0.2 \mathrm{~mm}$ \\
\hline $\mathrm{SiO}_{2}$ & $n=1.464$ & $k=0$ & $d=71.71 \mathrm{~nm}$ \\
\hline Substance-2 & $n=1.967$ & $k=0.00043$ & $d=11.15 \mathrm{~nm}$ \\
\hline Liquid & $n=1.34$ & &
\end{tabular}

Figure 9. Each surface of the plastic substrate designed with a double-layer anti-reflection coating in the liquid.

Tables 1 and 2 respectively show the transmission and reflection powers of the laser beam at the incident angles of $20^{\circ}$ and $45^{\circ}$ for ten as-deposited samples immersed in a liquid with a refraction index of 1.34. The transmittance and reflectance at a wavelength of $632.8 \mathrm{~nm}$ then are calculated with the baseline power of $3.08 \mathrm{~mW}$. We cannot measure the value at the normal incidence because of the layout of the measurement instruments. The average power of the transmission and the reflectance at a $20^{\circ}$ angle of incidence are $2.961 \mathrm{~mW}$ and $1.552 \mu \mathrm{W}$; those at $45^{\circ}$ are $2.936 \mathrm{~mW}$ and $11.16 \mu \mathrm{W}$, respectively. The transmittance and reflectance can be calculated by dividing the baseline values. Although the double-layer anti-reflection coating presents zero reflectance at only one wavelength and a low reflectance limit in only a narrow region, the average transmittances of the two incident angles are $96.13 \%$ and $95.32 \%$; and the average reflectances are only $0.050 \%$ and $0.362 \%$, respectively. As can be seen from Figure 1, the blurred red light passes through the round beaker due to the light scattering of the liquid. At incident angles of $20^{\circ}$ and $45^{\circ}$, the sum of the light scattering and the light absorption of the liquid are $3.813 \%$ and $4.31 \%$, respectively, and can be evaluated by subtracting the transmittance and reflectance. The reflectance increases as the angle of incidence increases due to the decrease in the phase thickness of each layer in the double layer [3]. At the same time, the minimum reflection spectral position is shifted to less than $632.8 \mathrm{~nm}$. It is presumed that the reflectance at a normal incidence will be less than $0.050 \%$. After the optical evaluation of the ten samples in the liquid, the double layers on the surfaces remain intact. Furthermore, the standard deviations of the reflectance of $0.050 \%$ and $0.362 \%$ shown in Tables 1 and 2 , are only $0.0023 \%$ and $0.0012 \%$, respectively. This indicates that the double-layer sample immersed in the liquid has very good optical stability. It is possible to speculate that the ion-assisted deposition in the deposition process solidifies the deposited film so that the moisture or water in the environment hardly penetrates the film and changes its refractive index $[10,17]$.

Table 1. The transmission and reflection of ten samples measured at an incident angle of $20^{\circ}$, where the baseline power of the He-Na laser beam equals $3.08 \mathrm{~mW}$.

\begin{tabular}{cccccc}
\hline Sample & $\boldsymbol{T}(\mathbf{m W})$ & $\boldsymbol{T}(\mathbf{\%})$ & $\boldsymbol{R}(\boldsymbol{\mu W})$ & $\boldsymbol{R}(\mathbf{\%})$ & Absorption and Scattering $(\mathbf{\%})$ \\
\hline 01 & 2.97 & 96.43 & 1.43 & 0.046 & 3.53 \\
02 & 2.95 & 95.78 & 1.49 & 0.048 & 4.17 \\
03 & 2.96 & 96.10 & 1.47 & 0.048 & 3.85 \\
04 & 2.96 & 96.10 & 1.51 & 0.049 & 3.85 \\
05 & 2.96 & 96.10 & 1.54 & 0.050 & 3.85 \\
06 & 2.97 & 96.43 & 1.61 & 0.052 & 3.52 \\
07 & 2.96 & 96.10 & 1.60 & 0.052 & 3.84 \\
08 & 2.96 & 96.10 & 1.61 & 0.052 & 3.84 \\
09 & 2.96 & 96.10 & 1.62 & 0.053 & 3.84 \\
10 & 2.96 & 96.10 & 1.64 & 0.053 & 3.84 \\
Average & 2.961 & 96.13 & 1.552 & $0.050 \pm 0.0023$ & 3.81 \\
\hline
\end{tabular}


Table 2. The transmission and reflection of ten samples measured at an incident angle of $45^{\circ}$, where the baseline power of the He-Na laser beam equals $3.08 \mathrm{~mW}$.

\begin{tabular}{cccccc}
\hline Sample & $\boldsymbol{T}(\mathbf{m W})$ & $\boldsymbol{T}(\mathbf{\%})$ & $\boldsymbol{R}(\boldsymbol{\mu W})$ & $\boldsymbol{R}(\mathbf{\%})$ & Absorption and Scattering $(\%)$ \\
\hline 01 & 2.94 & 95.45 & 11.13 & 0.361 & 4.18 \\
02 & 2.94 & 95.45 & 11.17 & 0.363 & 4.18 \\
03 & 2.94 & 95.45 & 11.17 & 0.363 & 4.18 \\
04 & 2.93 & 95.13 & 11.19 & 0.363 & 4.51 \\
05 & 2.93 & 95.13 & 11.14 & 0.362 & 4.51 \\
06 & 2.94 & 95.45 & 11.09 & 0.360 & 4.19 \\
07 & 2.93 & 95.13 & 11.20 & 0.364 & 4.51 \\
08 & 2.94 & 95.45 & 11.19 & 0.363 & 4.18 \\
09 & 2.93 & 95.13 & 11.18 & 0.363 & 4.51 \\
10 & 2.94 & 95.45 & 11.12 & 0.361 & 4.18 \\
Average & 2.936 & 95.32 & 11.16 & $0.362 \pm 0.0012$ & 4.31 \\
\hline
\end{tabular}

In this study, the two surfaces are designed to be in contact with liquid with refractive index of 1.43. The double-layer film design includes a low refractive index layer and then a high refractive index layer. If the upper surface is exposed to air, the admittance of the input surface should be designed to a value of one. In this case, the first layer may be designed using Substance- 2 with a high refraction index; and the second may be silicon dioxide with a low refraction index for the double-layer anti-reflection coating, using the above-mentioned method of the admittance diagram. Moreover, we can pre-deposit a $\mathrm{SiO}_{x}$ layer of several nanometers for the anti-reflection coating of the plastic substrate to enhance the adherence of the double layer [18].

As noted in the description of the experimental method above, the sample immured in the liquid is not conveniently measured by a conventional spectrometer. However, the theoretical reflectance of the two surfaces in the air without optical absorption of the substrate is $4.135 \%$ at a wavelength of $632.8 \mathrm{~nm}$ because the equivalent refractive index of each deposited double-layer surface is 1.34 [3]. Our other experiment in the air measured the reflectivity of about $4.223 \%$. The low reflectance of the sample in the liquid can also be approximately evaluated. However, the standard mean square error is $0.145 \%$, which is larger than $0.050 \%$ of the ultra-low reflectance measured in the liquid in the abovementioned experiment.

\section{Conclusions}

The reflectance of a plastic cover slip (Thermanox Coverslip-174977) immersed in the liquid with a refraction index of 1.34 for confocal image measurements was found to be about $0.05 \%$ at a wavelength of $632.8 \mathrm{~nm}$. The plastic cover slider can be used for biomedical applications of He-Na laser optical coupling due to its ultra-low reflectance. The plastic substrate, which had a low softening temperature, was only coated with double layers for anti-reflection properties. The coating was designed using the admittance diagram method, using starting materials of silicon monoxide granules for the first layer and Substance-2 for the second layer, wherein the first silicon dioxide layer was thicker due to the higher refractive index of the substrate. Moreover, plasma cleaning prior to deposition and ion-assisted deposition by an ion source under a non-heated condition facilitated the adhesion and optical stability of the deposited films in the liquid.

Acknowledgments: This work was financially supported by the Ministry of Science and Technology of Taiwan under Contract No. MOST 105-2221-E-030-007-MY3.

Author Contributions: Jin-Cherng Hsu and Heng-Ying Cho conceived and designed the experiments; Heng-Ying Cho performed the experiments; Tsang-Yen Hsieh and Jyh-Liang Wang analyzed the data; Jin-Cherng Hsu wrote the paper.

Conflicts of Interest: The authors declare no conflict of interest. 


\section{References}

1. Pawley, J.B. Handbook of Biological Confocal Microscopy, 3rd ed.; Springer Press: New York, NY, USA, 2007.

2. Guggenheim, E.J.; Lynch, I.; Rappoport, J.Z. Imaging In focus: Reflected light imaging: Techniques and applications. Int. J. Biochem. Cell Biol. 2017, 83, 65-70. [CrossRef] [PubMed]

3. Macleod, H.A. Thin-Film Optical Filters, 4th ed.; CRC Press: New York, NY, USA, 2010.

4. Apfel, J.H. Optical coating design with reduced electric field intensity. Appl. Opt. 1977, 16, 1880-1885. [CrossRef] [PubMed]

5. Arnon, O.; Baumeister, P. Electric field distribution and the reduction of laser damage in multilayers. Appl. Opt. 1980, 19, 1853-1855. [CrossRef] [PubMed]

6. Zhou, G.; Pun, C.F.J.; Tam, H.Y.; Wong, A.C.; Lu, C.; Wai, P.K.A. Single-mode perfluorinated polymer optical fibers with refractive index of 1.34 for biomedical applications. IEEE Photon. Technol. Lett. 2010, 22, 106-108. [CrossRef]

7. Hsu, J.C.; Lin, Y.H.; Wang, P.W.; Chen, Y.Y. Spectroscopic ellipsometry studies on various zinc oxide films deposited by ion beam sputtering at room temperature. Appl. Opt. 2012, 51, 1209-1215. [CrossRef] [PubMed]

8. Azzam, R.M.A.; Bashara, N.M.; Ballard, S.S. Ellipsometry and Polarized Light; North-Holland: Amsterdam, The Netherlands, 1977.

9. Kane, S.M.; Ahn, K.Y. Characteristics of ion-beam-sputtered thin films. J. Vac. Sci. Technol. 1979, 16, 171-174. [CrossRef]

10. Vallon, S.; Drevillon, B.; Poncin-Epaillard, F.; Klemberg-Sapieha, J.E.; Martinu, L. Argon plasma treatment of polycarbonate: In situ spectroellipsometry study and polymer characterizations. J. Vac. Sci. Technol. A 1996, 14, 3194-3201. [CrossRef]

11. Rao, K.N.; Mohan, S.; Hegde, M.S.; Balasubramanian, T.V. Chemical composition of electron-beam evaporated $\mathrm{TiO}_{2}$ films. J. Vac. Sci. Technol. A 1993, 11, 394-397. [CrossRef]

12. Catalán, L.A. Some computed optical properties of antireflection coatings. J. Opt. Soc. Am. 1962, 52, 437-440. [CrossRef]

13. Kahler, U.; Hofmeister, H. Visible light emission from Si nanocrystalline composites via reactive evaporation of SiO. Opt. Mater. 2001, 17, 83-86. [CrossRef]

14. Lee, C.C.; Hsu, J.C.; Jaing, C.C. Optical coatings on polymethyl methacrylate and polycarbonate. Thin Solid Films 1997, 295, 122-124. [CrossRef]

15. Lee, J.H.; Cho, J.S.; Koh, S.K.; Kim, D. Improvement of adhesion between plastic substrates and antireflection layers by ion-assisted reaction. Thin Solid Films 2004, 449, 147-151. [CrossRef]

16. Lee, C.C.; Hsu, J.C.; Wong, D.H. The characteristics of some metallic oxides prepared in high vacuum by ion beam sputtering. Appl. Surf. Sci. 2001, 171, 151-156. [CrossRef]

17. Hsu, J.C.; Wang, P.W.; Lin, Y.H.; Chen, H.L.; Chen, Y.Y.; Yao, Y.D.; Yu, J.C. Anti-reflective effect of transparent polymer by plasma treatment with end-Hall ion source and optical coating. Opt. Rev. 2010, 17, 553-556. [CrossRef]

18. Wendling, I.; Munzert, P.; Schulz, U.; Kaiser, N.; Tünnermann, A. Creating anti-reflective nanostructures on polymers by initial layer deposition before plasma etching. Plasma Process. Polym. 2009, 6, S716-S721. [CrossRef]

(C) 2017 by the authors. Licensee MDPI, Basel, Switzerland. This article is an open access article distributed under the terms and conditions of the Creative Commons Attribution (CC BY) license (http:/ / creativecommons.org/licenses/by/4.0/). 